\title{
Prevalence of virulence factors in enterotoxigenic Escherichia coli isolated from pigs with post-weaning diarrhoea in Europe
}

Andrea Luppi ${ }^{1 *}$, Mariavittoria Gibellini ${ }^{2+}{ }^{\dagger}$, Thomas Gin $^{3 \dagger}$, Frédéric Vangroenweghe ${ }^{4 \dagger}$, Virginie Vandenbroucke ${ }^{5}$, Rolf Bauerfeind ${ }^{6}$, Paolo Bonilauri ${ }^{1}, G^{\prime}$ Geoffrey Labarque ${ }^{3}$ and Álvaro Hidalgo ${ }^{7}$

\begin{abstract}
Background: Post-weaning diarrhoea (PWD), due to Escherichia coli, is an important cause of economic losses to the pig industry primarily as a result of mortality and worsened productive performance. In spite of its relevance, recent data about the prevalence of virulence genes and pathotypes among $E$. coli isolates recovered from cases of PWD in Europe are scarce.

Results: This study investigates the prevalence of fimbrial and toxin genes of $E$. coli by PCR among 280 farms with PWD across Europe. A total of 873 samples collected within the first $48 \mathrm{~h}$ after the onset of PWD (occurring 7-21 days post weaning) were submitted to the laboratory for diagnostic purposes. Isolation and identification of $E$. coli were performed following standard bacteriological methods and PCR assays for the detection of genes encoding for fimbriae (F4, F5, F6, F18 and F41) and toxins (LT, STa, STb and Stx2e). The prevalence of fimbriae and toxins among E. coli isolates from cases of PWD was: F4 (45.1\%), F18 (33.9 \%), F5 (0.6 \%), F6 (0.6 \%), F41 (0.3 \%), STb (59.1 \%), STa (38.1\%), LT (31.9\%) and Stx2e (9.7\%). E. coli isolates carrying both fimbrial and toxin genes were detected in 52. $5 \%$ of the cases (178 out of 339 isolates), with $94.9 \%$ of them being classified as enterotoxigenic E. coli (ETEC). The most common virotype detected was F4, STb, LT.
\end{abstract}

Conclusions: This study confirms that ETEC is frequently isolated in pig farms with PWD across Europe, with F4- and F18-ETEC variants involved in $36.1 \%$ and $18.2 \%$ of the outbreaks, respectively.

Keywords: Escherichia coli, Prevalence, ETEC, Post-weaning diarrhoea

\section{Background}

Post-weaning diarrhoea (PWD) in piglets remains a major cause of economic losses for the pig industry due to mortality, morbidity, decreased growth rate and cost of medication [1]. Enterotoxigenic Escherichia coli (ETEC) is regarded the most important cause of PWD, a pathotype characterized by the presence of fimbrial adhesins, which mediate attachment to porcine enterocytes, and enterotoxins, which disrupt fluid homeostasis in the small intestine. As a result of ETEC infection in

\footnotetext{
* Correspondence: andrea.luppi@izsler.it

${ }^{\dagger}$ Equal contributors

'Istituto Zooprofilattico Sperimentale della Lombardia e dell'Emilia Romagna (IZSLER), via Pitagora 2, Reggio Emilia, Italy

Full list of author information is available at the end of the article
}

PWD cases, mild to severe diarrhoea develops, typically within a few days after weaning, associated with dehydration, loss of body condition and increased mortality [2].

The adhesive fimbriae most commonly found in ETEC from pigs suffering PWD are those of types F4 (previously known as K88) and F18 (F107, 2134P, 8813). In addition, other adhesive fimbriae have been identified in E. coli isolates recovered from PWD cases, such as F5 (K99), F6 (987P), and F41, although more rarely [2-6]. The main enterotoxins detected in porcine ETEC are heat-labile toxin (LT), heat-stable toxin a (STa) and heatstable toxin b $(\mathrm{STb})$. Some strains can produce both enterotoxins and a Shiga toxin which is usually Stx2e 
subtype. Some authors classify these strains as ETEC rather than Shiga toxin producing E. coli (STEC) [2].

In spite of its relevance, only a few recent studies investigating the occurrence and the distribution of fimbriae and virulence factors among $E$. coli isolates from cases of PWD in Europe are available and in many cases they do not differentiate between $E$. coli isolates recovered from PWD cases and oedema disease or preweaning diarrhoea [4, 7]. Reports from Poland, Slovakia and Denmark suggest differences between countries among the prevalence of the main fimbrial types in E. coli isolates recovered from pigs with PWD $[4,6,8]$.

A simple and easy method to determine the presence of fimbrial adhesins of ETEC is slide agglutination. This method has been particularly useful for the identification of fimbrial adhesin F4, although is less reliable for other fimbria such as F6, F5 or F41 due to their variable expression in vitro [2,9]. Nowadays, genotypic analysis is commonly used to investigate the $E$. coli virotype involved in an infection and PCR genetic characterization is becoming increasingly available in veterinary diagnostic laboratories. Multiplex PCR for the detection of genes encoding for toxins (STa, STb, LT, and Stx2e) and fimbriae (F4, F5, F6, F18 and F41) is currently accessible to pig practitioners in Europe and can be an option for routine diagnostics of $E$. coli.

This study reports on the prevalence of virulence genes in enterotoxigenic $E$. coli isolates recovered from recent cases of PWD in pig farms across several countries in western and southern Europe.

\section{Methods}

\section{Sampling of pig farms with cases of PWD}

A total of 280 pig farms with acute cases of PWD were sampled as part of a diagnostic exercise between January 2012 and December 2014. Farms were located in the Flemish Region of Belgium and The Netherlands $(n=88)$, France $(n=91)$, Italy $(n=84)$ and Germany $(n=17)$. Sampled herds had a history of PWD occurring from 1 to 3 weeks after weaning, as evidenced by typical clinical signs: diarrhoea, decreased feed consumption, dehydration, depression and increased mortality.

Diagnostic specimens were obtained from 873 threeto five-week old pigs with diarrhoea (one sample per animal), within the first $48 \mathrm{~h}$ after the symptoms of PWD had occurred and before specific antimicrobial treatment had been administered. The use of zinc oxide in these outbreaks was not monitored. A detailed description of number and type of samples by country is presented in Table 1.

Isolation and characterization of Escherichia coli isolates Specimens were processed using standard procedures for isolation and characterization of intestinal E. coli [10].
Table 1 Number of farms, samples and sample types investigated in this study by country of origin

\begin{tabular}{llll}
\hline Country & Farms & \multicolumn{2}{l}{ Samples } \\
\cline { 3 - 4 } & $\mathrm{n}$ & $\mathrm{n}$ & Type $^{\mathrm{a}}$ \\
\hline Belgium and The Netherlands & 88 & 160 & $\mathrm{~S}, \mathrm{P}$ \\
France & 91 & 455 & $\mathrm{~S}$ \\
Germany & 17 & 99 & $\mathrm{~S}$ \\
Italy & 84 & 159 & S, F, I, P \\
Total & 280 & 873 & \\
\hline
\end{tabular}

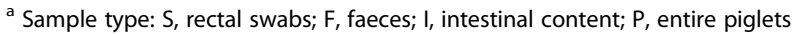

Briefly, samples were plated on selective media and on tryptose soy agar (TSA) medium supplemented with $5 \%$ of defibrinated ovine blood and incubated aerobically overnight at $37^{\circ} \mathrm{C}$. Haemolytic activity was evaluated and single coliform colonies were further characterized by biochemical methods.

\section{DNA extraction and characterization of virulence factors by PCR}

DNA samples were prepared from one up to five haemolytic and/or not haemolytic E. coli colonies and used to perform a multiplex PCR for the detection of fimbrial and toxin genes, including those encoding for $\mathrm{F} 4$ (K88), F5 (K99), F6 (987P), F18, F41, LT, STa, STb and Stx2e, but not discriminating between F4ab, F4ac and F4ad. The methodology used for the identification of these virulence genes has been described previously [11].

When $E$. coli isolates with the same combination of virulence factors (virotype) were detected in the same herd they were considered duplicates of an E. coli prototype strain. The prototype but not the duplicates were considered for prevalence calculations. E.coli strains carrying both fimbrial and toxin genes were classified into pathotypes. Isolates encoding at least one of the investigated enterotoxins together with Stx2e and F18 fimbriae were classified as ETEC based on the PWD clinical signs observed, as previously proposed [2]. Isolates carrying genes for adhesive fimbriae and Stx2e were classified as STEC.

\section{Results}

A total of 844 DNA samples belonging to the same number of $E$. coli isolates and representing all of the 280 PWD-affected pig farms included in this study were analysed by PCR. These isolates were classified as being positive or negative for the presence of one or more virulence genes (F4, F5, F6, F18, F41, LT, STa, STb and Stx2e). Following these criteria and excluding isolates which were considered duplicates within herds, the prevalence of virulence genes was calculated. Eighty nine per cent (302 out of 339) of E. coli isolates carried at 
least one virulence gene, while $10.9 \%$ (37 out of 339) resulted negative for all of the virulence genes investigated. Fimbrial genes were identified in $75.8 \%$ (257 out of 339) and toxin genes in $65.8 \%$ (223 out of 339) of the isolates. In 28.3 \% (96 out of 339) of the E. coli isolates, a single virulence gene was detected.

The prevalence of fimbrial and toxin genes in nonduplicate E. coli isolates from PWD-affected pig farms across Europe is shown in Table 2. Overall, the adhesive fimbriae most commonly detected was F4 (45.1\%), followed by F18 (33.9 \%). Sixteen isolates possessed genes for two types of fimbriae. The combination of $\mathrm{F} 4$ and F18 was detected in 15 isolates, with and without toxin genes, whereas F5 and F41 in one isolate. The most prevalent toxin was STb (59.1\%), followed by STa (38.1\%) and LT (31.9\%).

Based on the presence of genes for both fimbriae and toxins, $52.5 \%$ (178 out of 339) of the E. coli isolates were classified into pathotypes (Table 3 ). Within these, ETEC was the most frequent pathotype with $94.9 \%$ of the isolates (169 out of 178). The ETEC virotypes most commonly detected were (i) F4, STb, LT; (ii) F4, STa, STb, LT and (iii) F4, STa, STb, found in 49, 28 and 18 isolates, respectively. Adhesive fimbria F18 was mainly detected in combination with genes encoding for (i) STa, STb and (ii) STa, STb, Stx2e. Among the ETEC, 23 isolates encoded for Stx2e in addition to enterotoxins and fimbriae. Nine isolates were classified as STEC, harbouring only genes for F18 and Stx2e.

E.coli characterized as ETEC were haemolytic in $97.6 \%$ of the cases. The remaining $2.4 \%$ nonhaemolytic ETEC isolates, for which haemolytic activity was consistently tested, were recovered in France, Italy and Germany, sharing the same virotype: F4, STa, STb. All STEC isolates (F18, Stx2e) were haemolytic.

ETEC was found in $59.6 \%$ of the 280 PWD-affected farms investigated across Europe. F4-ETEC was the main fimbrial type, being identified as single virotype in $36.1 \%$ of the farms. F18-ETEC was isolated in $18.2 \%$ of the farms. In $4.6 \%$ of the farms, an enterotoxigenic
E. coli virotype harbouring both F4 and F18 fimbriae was found. Two different fimbrial subtypes of ETEC were identified in one farm, F4-ETEC (F4, STa, STb, LT) and F18-ETEC (F18, STa, STb). An itemization of ETEC prevalence among farms with PWD by country is shown in Table 4.

\section{Discussion}

The present study describes the prevalence of fimbrial and toxin genes detected in E. coli isolated from piglets in the early stages of PWD across Belgium, The Netherlands, France, Germany and Italy. From the different adhesin fimbriae investigated, F4 was the type most commonly detected, followed by F18 (45.1\% and $33.9 \%$ of the isolates, respectively). Similarly, a higher prevalence of F4 than F18 fimbriae has been reported before in other European countries including Denmark [4], Slovakia [12] and Czech Republic [13]. Nevertheless, data from Slovakia and Poland have shown a higher prevalence of F18 fimbriae [6, 14]. Outside Europe, a higher prevalence of F18-ETEC has been reported in Cuban pigs with diarrhoea [15], while F4 was the most common fimbriae associated to the prevalent E.coli serogroups (O149 and O141) causing diarrhoea in Australian pigs [16]. The F4 fimbrial gene was reported in $64.6 \%$ of E.coli strains isolated from pigs with diarrhoea in the United States [17].

A small number of isolates containing F5, F6 and F41 genes were also found in our study. Although these adhesive fimbriae are usually found in $E$. coli isolates recovered from younger pigs, its detection in isolates obtained from post-weaning pigs has been described previously [3-6]. Regarding enterotoxins, the most prevalent one was STb (59.1\% of isolates), usually associated to a severe fluid loss in the small intestine of weaned piglets. The prevalence of STb is in accordance with previous studies $[4,17]$.

In $52.5 \%$ of the isolates, a combination of fimbriae and toxins was detected and isolates were classified accordingly into recognized pathotypes [2]. Two pathotypes,

Table 2 Prevalence of examined genes for fimbriae and toxins among 339 isolates of E. coli recovered from pigs with PWD across Europe

\begin{tabular}{|c|c|c|c|c|c|c|c|c|c|}
\hline \multirow[t]{3}{*}{ Country and number of isolates tested } & \multicolumn{9}{|c|}{ Percentage (number) of positive E. coli isolates ${ }^{a}$} \\
\hline & \multicolumn{5}{|l|}{ Fimbriae } & \multicolumn{4}{|l|}{ Toxins } \\
\hline & F4 & F5 & F6 & F18 & F41 & LT & STa & STb & Stx2e \\
\hline Belgium and The Netherlands $(n=100)$ & $51.0(51)$ & $1.0(1)$ & $1.0(1)$ & $42.0(42)$ & - & $14.0(14)$ & $22.0(22)$ & $30.0(30)$ & $5.0(5)$ \\
\hline France $(n=91)$ & $47.3(43)$ & - & - & $35.2(32)$ & - & $45.1(41)$ & $40.7(37)$ & $76.9(70)$ & $19.8(18$ \\
\hline Germany $(n=64)$ & $14.1(9)$ & - & - & $14.1(9)$ & - & $9.4(6)$ & $26.6(17)$ & $57.8(37)$ & $3.1(2)$ \\
\hline Italy $(n=84)$ & $59.3(50)$ & $1.2(1)$ & $1.2(1)$ & $38.1(32)$ & $1.2(1)$ & $56.0(47)$ & $63.1(53)$ & $71.4(60)$ & $9.5(8)$ \\
\hline All countries $(n=339)$ & $45.1(153)$ & $0.6(2)$ & $0.6(2)$ & $33.9(115)$ & $0.3(1)$ & 31.9 (108) & $38.1(129)$ & $59.1(197)$ & $9.7(33)$ \\
\hline
\end{tabular}

${ }^{a}$ Isolates carrying genes for two fimbriae or two or more toxins were identified in 16 and 178 cases respectively 
Table 3 Distribution of encoded virulence factor combinations among 178 E. coli isolates from European cases of PWD classified as ETEC or STEC in decreasing order of prevalence

\begin{tabular}{|c|c|c|c|c|c|c|}
\hline \multirow[t]{3}{*}{ Virulence factor combination } & \multicolumn{6}{|c|}{ Number of positive E. coli isolates } \\
\hline & \multirow{2}{*}{$\begin{array}{l}\text { Belgium and The Netherlands } \\
\mathrm{n}\end{array}$} & \multirow{2}{*}{$\begin{array}{l}\text { France } \\
\mathrm{n}\end{array}$} & \multirow{2}{*}{$\begin{array}{l}\text { Italy } \\
\mathrm{n}\end{array}$} & \multirow{2}{*}{$\begin{array}{l}\text { Germany } \\
\mathrm{n}\end{array}$} & \multicolumn{2}{|c|}{ All countries } \\
\hline & & & & & $n$ & $\%$ \\
\hline F4, STb, LT & 7 & 21 & 17 & 4 & 49 & 27.5 \\
\hline F4, STa, STb, LT & 3 & 10 & 15 & - & 28 & 15.7 \\
\hline F4, STa, STb & 9 & 2 & 3 & 4 & 18 & 10.1 \\
\hline F18, STa, STb & 7 & 6 & 3 & - & 16 & 9.0 \\
\hline F18, STa, STb, Stx2e & 1 & 6 & 8 & 1 & 16 & 9.0 \\
\hline F18, Stx2e & 4 & 4 & - & 1 & 9 & 5.1 \\
\hline F18, STa, STb, LT & - & - & 7 & - & 7 & 3.9 \\
\hline F18, STa & 1 & - & 5 & - & 6 & 3.4 \\
\hline F18, STb, LT & 1 & 2 & - & - & 3 & 1.7 \\
\hline F4, F18, STa, STb, LT & - & 1 & 2 & - & 3 & 1.7 \\
\hline F4, F18, STa, STb, Stx2e & - & 3 & - & - & 3 & 1.7 \\
\hline $\mathrm{F} 4, \mathrm{LT}$ & 3 & - & - & - & 3 & 1.7 \\
\hline F18, STa, LT & - & - & 2 & - & 2 & 1.1 \\
\hline F4, F18, STa, STb & - & 2 & - & - & 2 & 1.1 \\
\hline $\mathrm{F} 4, \mathrm{STa}$ & - & - & 2 & - & 2 & 1.1 \\
\hline $\mathrm{F} 4, \mathrm{STb}$ & - & - & 2 & - & 2 & 1.1 \\
\hline F18, STb & - & 1 & - & - & 1 & 0.6 \\
\hline F18, STb, LT, Stx2e & - & 1 & - & - & 1 & 0.6 \\
\hline F4, F18, STa & - & - & 1 & - & 1 & 0.6 \\
\hline F4, F18, STa, LT & - & - & 1 & - & 1 & 0.6 \\
\hline F4, F18, STb, LT & - & 1 & - & - & 1 & 0.6 \\
\hline F4, F18, STb, LT, Stx2e & - & 1 & - & - & 1 & 0.6 \\
\hline F4, F18, STa, STb, LT, Stx2e & - & 1 & - & - & 1 & 0.6 \\
\hline F4, STa, STb, LT, St×2e & - & 1 & - & - & 1 & 0.6 \\
\hline $\mathrm{F} 5, \mathrm{STb}$ & 1 & - & - & - & 1 & 0.6 \\
\hline
\end{tabular}

ETEC and STEC, were found among E. coli isolates from cases of PWD in Europe, with the vast majority being classified as ETEC (94.9\%). The high recovery rate in our study is possibly influenced by the sampling being conducted in the first $48 \mathrm{~h}$ after the onset of PWD and before any antibiotic treatment and thus increasing the likelihood of detecting the causal agent rather than other resident E. coli.

In a small number of isolates $\mathrm{F} 4$ and F18 fimbrial genes were detected concurrently (F4, F18-ETEC).

Table 4 Prevalence of ETEC fimbrial subtypes among 280 pig farms with cases of PWD examined in Europe

\begin{tabular}{llllll}
\hline ETEC subtype & \multicolumn{4}{l}{ Percentage $^{\text {a }}$ (number) of affected pig farms in a given country } \\
\cline { 2 - 5 } & \multicolumn{2}{l}{ Belgium and The Netherlands } & France & Italy & Germany $^{\text {All countries }}$ \\
\hline F4-ETEC & $23.9(21)$ & $37.4(34)$ & $46.4(39)$ & $41.2(7)$ & $36.1(101)$ \\
F18-ETEC & $10.2(9)$ & $17.6(16)$ & $29.8(25)$ & $5.9(1)$ & $18.2(51)$ \\
F4,F18-ETEC & - & $9.9(9)$ & $4.8(4)$ & - & $4.6(13)$ \\
F5-ETEC & $1.1(1)$ & - & - & - & $0.4(1)$ \\
F4-ETEC \& F18-ETEC & $1.1(1)$ & - & - & - & $0.4(1)$ \\
All subtypes & $36.4(32)$ & $64.8(59)$ & $81.0(68)$ & $47.1(8)$ & $59.6(167)$
\end{tabular}

a Percentage of positive farms over total farms investigated in a given country: Belgium and The Netherlands, 88; France, 91; Italy, 84 and Germany, 17

${ }^{b}$ Percentage of positive farms over all farms investigated $(n=280)$ 
Whereas less frequent than other subtypes, ETEC isolates encoding for more than one of the examined adhesive fimbriae have been described before $[3-5,12]$. ETEC strains possessing multiple adhesins could have a pathogenetic advantage, as supposed previously [18].

In the present study the fimbrial F4 gene was strongly associated with LT, STb and with LT, STa, STb enterotoxin gene combinations (49 and 28 isolates, respectively), which is in line with a previous report from Denmark [4]. Similar results have been described concerning the association of F4 with LT and STb, but not with STa in the United States and Slovakia [6, 17]. Interestingly, a drastic increase in the prevalence of STa has been reported in Canada when F4-ETEC isolates from 1998-2001 were compared with 1974-1987 isolates [19]. Whereas our data does not allow us to make any inference about the recent emergence of an F4-ETEC virotype containing the STa gene in Europe, it does suggest geographical differences among the major virotypes of F4-ETEC detected in cases of PWD. In addition, further genetic characterization is required to determine if the high prevalence of some virotypes identified in this study is the result of clonal expansion or horizontal gene transfer.

The third most frequent combination of fimbrial and toxin genes was F4, STa, STb, with 18 isolates. It is noteworthy that four non-haemolytic ETEC isolates detected in our study belonged to this virotype. These isolates originated from farms geographically dispersed (France, Italy and Germany), indicating that non-haemolytic ETEC can play a role in some of the PWD outbreaks in agreement with previous findings [4, 17]. The haemolytic phenotype is frequently used as an indication of $E$. coli pathogenicity among isolates recovered from PWD cases. However, non-haemolytic isolates, even if uncommon, should not be discarded in the diagnostic process particularly when clinical and pathological findings are indicative of PWD.

Twenty eight per cent of the isolates encoded for a single virulence factor and $10.9 \%$ lacked any of the virulence genes investigated, being considered nonpathogenic. Isolates carrying at least a toxin without any known fimbriae and vice versa have been already described $[6,20]$. The possible role of these strains in the development of PWD may require further investigation in order to evaluate the presence of other virulence factors such as adhesins or enterotoxins that may be involved in the pathogenesis of the disease. For example, AIDA (adhesin involved in diffuse adherence) has been associated with $E$. coli strains recovered from piglets with diarrhoea, and there is evidence that it is causatively involved in diarrhoea experimentally induced in colostrum-deprived newborn piglets with STb encoding E. coli [21]. However, evidence for any effect in elder or conventionally reared piglets is still lacking. Similarly, the toxin EAST1 (enteroaggregative E. coli heat stable enterotoxin) is commonly detected among porcine ETEC although its role in PWD remains yet to be clarified $[13,22]$. Likewise, we cannot exclude the presence of enteropathogenic E. coli (EPEC) among those isolates or the involvement of other pathogens in the cases of PWD described in this study. EPEC can also cause PWD in pigs although it does not possess any of the classic virulence factors of ETEC [2].

In this study, 167 of the farms investigated (59.6\%) were positive for the presence of ETEC in samples from pigs with diarrhoea. Moreover, the examination of ETEC fimbrial subtypes revealed that F4-ETEC is the main subtype associated with PWD cases in Europe, followed by F18-ETEC. A higher prevalence of PWD-affected farms with F4-ETEC compared to F18-ETEC was consistently shown in all countries included in this study, with approximately twice as many outbreaks being associated with F4-ETEC as with F18-ETEC. In spite of its clinical relevance, the prevalence of pathotypes and subtypes associated with PWD outbreaks is not usually reported as a proportion of clinical cases at farm level, but as percentage of isolates investigated $[3,8,12,13]$.

\section{Conclusions}

Our study confirms that ETEC is the main pathotype involved in clinical cases of PWD in European pig farms and that F4-ETEC is the main fimbrial subtype, followed by F18-ETEC.

The information presented in this study has practical relevance for the control of PWD in European pig producing systems. A correct sampling of diseased pigs, laboratory confirmation of the aetiological agent and associated virulence factors are required in order to achieve an early diagnosis and understand the role of $E$. coli at the onset of PWD outbreaks.

\section{Abbreviations \\ AIDA, adhesin involved in diffuse adherence; EAST1, enteroaggregative E. coli heat stable enterotoxin; EPEC, enteropathogenic E.coli; ETEC, enterotoxigenic E.coli; LT, heat-labile toxin; PWD, post-weaning diarrhoea; STa, heat-stable toxin a; STb, heat-stable toxin b; STEC, Shiga toxin-producing E. coli; Stx2e, Shiga toxin subtype 2e; TSA, tryptose soy agar}

\section{Acknowledgements \\ The authors would like to express their gratitude to Marc Henninger (Elanco Animal Health, France) and to the practitioners that contributed to the sampling of pigs across Europe for this study. This study was financed by Elanco Animal Health.}

Funding

"Not applicable".

Availability of data and material "Not applicable". 


\section{Authors' contributions}

AL performed laboratory diagnostics, analysed the data and assisted in drafting the manuscript. MG, TG, FV visited the farms, collected the samples and interpreted the data. W performed the laboratory diagnostics and analysed the data. RB and PB performed the laboratory diagnostics, analysed the data and critically reviewed the manuscript. GL participated in the conception and design of the study and critically reviewed the manuscript $\mathrm{AH}$ participated in the design of the study and interpretation of the data and drafted the manuscript. All authors read and approved the final manuscript.

\section{Competing interests}

Mariavittoria Gibellini, Thomas Gin, Frédéric Vangroenweghe, Geoffrey Labarque and Álvaro Hidalgo are employees of Elanco Animal Health. Andrea Luppi and Rolf Bauerfeind have temporarily collaborated as external experts in the swine advisory board of Elanco Animal Health.

\section{Consent for publication}

"Not applicable".

\section{Ethics approval and consent to participate}

"Not applicable".

\begin{abstract}
Author details
'Istituto Zooprofilattico Sperimentale della Lombardia e dell'Emilia Romagna (IZSLER), via Pitagora 2, Reggio Emilia, Italy. ${ }^{2}$ Elanco Animal Health, Divisione veterinaria Eli Lilly Italia Spa, Via A. Gramsci, 731, 50019 Sesto Fiorentino, FI, Italy. ${ }^{3}$ Elanco Animal Health, 24 Boulevard Vital Bouhot, 92521

Neuilly-sur-Seine, France. ${ }^{4}$ Elanco Animal Health Benelux, Plantijn en Moretuslei 1 -3rd floor, 2018 Antwerpen, Belgium. ${ }^{5}$ Dierengezondheidszorg (DGZ) Vlaanderen, Industrielaan 29, 8820 Torhout, Belgium. ${ }^{6}$ Institute of Hygiene and Infectious Diseases of Animals, Justus Liebig University, Frankfurter Str. 85-89, 35392 Giessen, Germany. ${ }^{7}$ Elanco Animal Health, Lilly House, Priestley Road, Basingstoke RG249NL, UK.
\end{abstract}

Received: 30 March 2016 Accepted: 15 July 2016

Published online: 01 September 2016

\section{References}

1. Fairbrother JM, Nadeau É, Gyles CL. Escherichia coli in postweaning diarrhea in pigs: an update on bacterial types, pathogenesis, and prevention strategies. Anim Health Res Rev. 2005;6:17-39.

2. Fairbrother JM, Gyles CL. Colibacillosis. In: Zimmerman JJ, Karriker LA, Ramirez A, Schwartz KJ, Stevenson GW, editors. Disease of Swine. 10th ed. UK: Wiley-Blackwell; 2012. p. 723-47.

3. Kwon D, Choi C, Jung T, Chung HK, Kim JP, Bae SS, Cho WS, Kim J, Chae C. Genotypic prevalence of the fimbrial adhesins (F4, F5, F6, F41 and F18) and toxins (LT, STa, STb and Stx2e) in Escherichia coli isolated from postweaning pigs with diarrhea or edema disease in Korea. Vet Rec. 2002;150:35-7.

4. Frydendahl K. Prevalence of serogroups and virulence genes in $E$. coli associated with postweaning diarrhoea and edema disease in pigs and a comparison of diagnostic approaches. Vet Microbiol. 2002;85:169-82.

5. Chen X, Gao S, Jiao X, Xiu FL. Prevalence of serogroups and virulence factors of $E$. coli strains isolated from pigs with postweaning diarrhoea in eastern China. Vet Microbiol. 2004;103:13-20.

6. Vu-Khac H, Holoda E, Pilipcinec E, Blanco M, Blanco JE, Mora A, Dahbi G, López C, González EA, Blanco J. Serotypes, virulence genes, and PFGE profiles of Escherichia coli isolated from pigs with postweaning diarrhea in Slovakia. BMC Vet Res. 2006:2:10.

7. Garabal Jl, Vázquez F, Blanco J, Blanco M, González EA. Colonization antigens of enterotoxigenic Escherichia coli strains isolated from piglets in Spain. Vet Microbiol. 1997;54:321-8.

8. Osek J. Prevalence of virulence factors of Escherichia coli strains isolated from diarrheic and healthy piglets after weaning. Vet Microbiol. 1999;68:209-17.

9. Mullaney CD, Francis DH, Willgohs JA. Comparison of seroagglutination ELISA, and indirect fluorescent antibody staining for the detection of K99, K88, and 987P pilus antigens of Escherichia coli. J Vet Diagn Invest. 1991;3:115-8.
10. Luppi A, Bonilauri P, Dottori M, Gherpelli Y, Biasi G, Merialdi G, Maioli G, Martelli P. Antimicrobial resistance of F4+ Escherichia coli isolated from Swine in Italy. Transbound Emerg Dis. 2013;62:67-71.

11. Casey TA, Bosworth BT. Design and evaluation of a multiplex polymerase chain reaction assay for the simultaneous identification of genes for nine different virulence factors associated with Escherichia coli that cause diarrhea and edema disease in swine. J Vet Diagn Invest. 2009;21:25-30.

12. Vu-Khac H, Holoda E, Pilipcinec E. Distribution of virulence genes in E. coli strains isolated from diarrhoeic piglets in the Slovak Republic. J Vet Med B Infect Dis Vet Public Health. 2004;51:343-7.

13. Zajacova ZS, Konstantinova L, Alexa P. Detection of virulence factors of Escherichia coli focused on prevalence of EAST1 toxin in stool of diarrheic and non-diarrheic piglets and presence of adhesion involving virulence factors in astA positive strains. Vet Microbiol. 2012;154:369-75.

14. Osek J, Gallien P. Truszczyñski M, Protz D. The use of polymerase chain reaction for determination of virulence factors of Escherichia coli strains isolated from pigs in Poland. Comp Immunol Microbiol Infect Dis. 1999;22:163-74.

15. Blanco M, Lazo L, Blanco JE, Dahbi G, Mora A, López C, González EA, Blanco J. Serotypes, virulence genes, and PFGE patterns of enteropathogenic Escherichia coli isolated from Cuban pigs with diarrhea. Int Microbiol. 2006;1:53-60.

16. Smith MG, Jordan D, Chapman TA, Chin JJ, Barton MD, Do TN, Fahy VA, Fairbrother JM, Trott DJ. Antimicrobial resistance and virulence gene profiles in multi-drug resistant enterotoxigenic Escherichia coli isolated from pigs with post-weaning diarrhoea. Vet Microbiol. 2010;145:299-307.

17. Zhang W, Zhao M, Ruesch L, Omot A, Francis D. Prevalence of virulence genes in Escherichia coli strains recently isolated from young pigs with diarrhea in the US. Vet Microbiol. 2007;123:145-52.

18. Nagy B, Fekete PZ. Enterotoxigenic Escherichia coli (ETEC) in farm animals. Vet Res. 1999;30:259-84.

19. Noamani BN, Fairbrother JM, Gyles CL. Virulence genes of O149 enterotoxigenic Escherichia coli from outbreaks of postweaning diarrhea in pigs. Vet Microbiol. 2003;97:87-101.

20. Nagy B, Casey TA, Moon HW. Phenotype and genotype of Escherichia coli isolated from pigs with postweaning diarrhoea in Hungary. J Clin Microbiol. 1990;28:651-3.

21. Ravi M, Ngeleka M, Kim SH, Gyles C, Berthiaume F, Mourez M, Middleton D, Simko E. Contribution of AIDA-I to the pathogenicity of a porcine diarrheagenic Escherichia coli and to intestinal colonization through biofilm formation in pigs. Vet Microbiol. 2007;120:308-19.

22. Zajacova ZS, Faldyna M, Kulich P, Kummer V, Maskova J, Alexa P. Experimental infection of gnotobiotic piglets with Escherichia coli strains positive for EAST1 and AIDA. Vet Immunol Immunopathol. 2013;152:176-82.

\section{Submit your next manuscript to BioMed Central and we will help you at every step:}

- We accept pre-submission inquiries

- Our selector tool helps you to find the most relevant journal

- We provide round the clock customer support

- Convenient online submission

- Thorough peer review

- Inclusion in PubMed and all major indexing services

- Maximum visibility for your research

Submit your manuscript at www.biomedcentral.com/submit
) Biomed Central 